\title{
Parto y aborto en tiempos de coronavirus: el impacto de la pandemia en los derechos sexuales y reproductivos*
}

\author{
Ester Massó Guijarro \\ Universidad de Granada. FiloLab \\ ester@ugr.es
}

\section{Rosana Triviño Caballero}

Universidad de Alcalá. Departamento de Cirugía, Ciencias Médicas y Sociales rosanatrivino@gmail.com

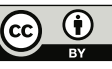

Fecha de recepción: 15-6-2020

Fecha de aceptación: 30-6-2020

\section{Resumen}

Los derechos sexuales y reproductivos de las mujeres y la primera infancia se encuentran amenazados debido a la crisis ocasionada por la COVID-19. Tanto las mujeres gestantes y puérperas como sus criaturas están siendo víctimas de las consecuencias del paradigma del patriarcado ginecobstétrico en tiempos de pandemia. En este artículo analizamos las consecuencias negativas que ha tenido la gestión del coronavirus en los procesos de parto y aborto. Asimismo, defendemos que la crisis desencadenada por la COVID-19 puede ser una oportunidad para visibilizar situaciones de injusticia sexual y reproductiva y para provocar cambios que garanticen los derechos y las libertades de gestantes y criaturas.

Palabras clave: aborto; aborto farmacológico; confinamiento; COVID-19; derechos sexuales y reproductivos; distanciamiento social; gestación; parto; patriarcado obstétrico

\section{Abstract. Childbirth and abortion in times of coronavirus: The impact of the pandemic on sexual and reproductive rights}

The sexual and reproductive rights of women, as well as the rights of their children, are being threatened due to the COVID-19 crisis. In these times of pandemic, both pregnant and postpartum women and their newborns have become victims of the consequences of the gyno-obstetric patriarchy paradigm. In this article, we analyze the negative consequences of coronavirus management in childbirth and abortion processes. Likewise, we defend that the crisis unleashed by COVID-19 can be an opportunity to make visible situations of sexual and reproductive injustice and bring about changes to guarantee the freedoms and rights of women and infants.

Keywords: abortion; pharmacological abortion; confinement; COVID-19; sexual and reproductive rights; social distancing; pregnancy; childbirth; obstetric patriarchy

* Deseamos agradecer al maravilloso equipo de ESPACyOS (Ética Salubrista para la Acción, Cuidado y Observación Social) el haber sido motor pionero de inspiración para estas reflexiones. Hay una mención especial también para las revisoras, cuya labor sin duda mejoró sustancialmente el manuscrito original. 


\section{Sumario}

1. Introducción: agencia reproductiva en tiempos de pandemia, ¿lujo o derecho?

2. El aborto en tiempos de coronavirus
4. Conclusiones

Referencias bibliográficas

3. El parto en tiempos de coronavirus

\section{Introducción: agencia reproductiva en tiempos de pandemia, ¿lujo o derecho?}

La inédita y radical transformación que las sociedades humanas están experimentando desde la generalización de la enfermedad por coronavirus ha impactado de modo significativo en los derechos y en las libertades de las mujeres y sus criaturas. Tanto las gestantes y puérperas como la primera infancia están siendo víctimas del paradigma del patriarcado obstétrico ${ }^{1}$ y su específica biopolítica en tiempos de pandemia. En este artículo presentamos un análisis preliminar sobre el derecho a procesos de parto y aborto ${ }^{2}$ autónomos y respetados, que se han visto vulnerados específicamente desde el advenimiento de la pandemia. Defenderemos que esta reacción expresa la falta de reconocimiento de la agencia de las mujeres gestantes, puesto que las medidas adoptadas no responden a necesidades de salud pública, sino a inercias asistenciales y a intereses ideológicos. Desde nuestro punto de vista, la gestión de la crisis puede ser una oportunidad para visibilizar situaciones de injusticia sexual y reproductiva, analizar falsas creencias y revisar la evidencia científica en relación con el parto y el aborto, con el fin de garantizar la libertad de elección de las mujeres y los derechos de la primera infancia.

En referencia al embarazo, los protocolos en España sobre la limitación del acompañamiento a las mujeres durante el parto en la actual situación de pandemia han motivado graves denuncias por atentar contra los derechos humanos, la legislación vigente y la evidencia científica ${ }^{3}$. Protestas, manifiestos y

1. Ver su definición, su acotación y sus extensiones en Almaguer González et al. (2010) e incluso se sugiere regresar al texto de Campbell et al. (1997), que, pese a su ya cierta antigüedad, continúa plenamente vigente.

2. El término aborto incluye diferentes formas de interrupción del embarazo. Para el propósito de este trabajo, estará fundamentalmente referido a la finalización intencionada de la gestación en cualquiera de sus estadios y será utilizado indistintamente junto con las expresiones interrupción del embarazo o interrupción de la gestación. Se ha evitado conscientemente el uso de la forma interrupción voluntaria del embarazo, utilizada en la regulación legal de la práctica, en la medida en que el matiz de voluntariedad puede connotar que se trata de una modalidad no prioritaria o esencial, que es precisamente la idea que se pretende cuestionar en este trabajo. En relación con esta cuestión, resulta de interés el trabajo de Katie Watson titulado "Why We Should Stop Using the Term "Elective Abortion" (2018).

3. Para saber más sobre su naturaleza, su importancia radical y cómo se regula, ver, por ejemplo: Tarodo y González de Zárate (2012: 273 y 275), Rámila et al. (2016: 9 s.), e incluso el artículo 8 del convenio europeo de Derechos Humanos, que establece el derecho al 
estudios especializados concuerdan en visibilizar el impacto de la crisis provocada por la COVID-19 sobre la justicia sexual y reproductiva en su dimensión interseccional (Ross, 2017).

En referencia al aborto, por un lado, los desplazamientos requeridos para acceder a la interrupción del embarazo resultan contradictorios con la minimización de la exposición al virus; por otro lado, la crisis de la COVID-19 ha parapetado iniciativas ideológicas encaminadas a restringir el acceso al aborto, con frecuencia bajo la premisa de que se trata de una prestación no esencial (Guttmatcher Institute, 2020; Hussein, 2020). Ante semejante situación, se ha reivindicado que las gestiones obligatorias se puedan realizar de manera telemática y que sea posible acceder de forma segura al aborto farmacológico (Robinson et al., 2020). Asimismo, se ha apelado a la necesidad de que la COVID-19 no se convierta en una excusa para vulnerar el derecho al aborto y obstaculizar su ejercicio.

Se plantea, en definitiva, la necesidad de reflexionar sobre la justicia en torno a la salud sexual y reproductiva, todo ello en un marco epistémico que incluye las implicaciones de las dimensiones de género en relación con el virus. Afirmamos, pues, con McGovern et al. (2020), que las cuestiones de salud sexual y reproductiva no pueden ser consideradas «un lujo», una especie de «extra», tampoco en tiempos de pandemia. Su condición esencialmente femenina, de acuerdo con el paradigma del patriarcado obstétrico (Almaguer et al., 2010; Campbell et al., 1997), da lugar a que se releguen frente a asuntos considerados menos «específicos», más relevantes, aunque todo lo vinculado a la reproducción sea fundamental para la humanidad en su integridad.

\section{El aborto en tiempos de coronavirus}

En lugares donde el acceso al aborto está legalmente reconocido, las dificultades a las que se enfrentan las mujeres embarazadas para conseguirlo son abundantes, también en la era previa a la COVID-19. Los procesos administrativos de duración variable, la objeción de conciencia del personal médico, la institucionalización de la práctica en su modalidad farmacológica o la necesidad de viajar a otros países o regiones son obstáculos frecuentes para las mujeres que desean abortar (Sethna y Davis, 2019; Triviño, 2018).

El impacto de estos problemas se agrava en el contexto actual. La pandemia provocada por la COVID-19 ha incrementado las restricciones en el acceso a las medidas de contracepción y al aborto en todo el mundo (IPPF, 2020). Ciertamente, dichas prestaciones no son las únicas que han sufrido el impacto de esta crisis. Su especificidad se concreta en varias dimensiones. En primer lugar, se trata de procedimientos en los que el retraso en el acceso a los recursos necesarios puede suponer la imposibilidad legal de interrumpir el embarazo.

respeto de la vida privada y familiar, impidiendo a las autoridades públicas intervenir en dicho ámbito a menos que la ley así lo contemple (<https://www.derechoshumanos.net/ Convenio-Europeo-de-Derechos-Humanos-CEDH/articulo8CEDH.htm>). 
En segundo lugar, las consecuencias de estas restricciones tienen una importante incidencia en la salud, la autonomía y la vida de muchas mujeres y su entorno, en especial sobre aquellas que se encuentren en especial situación de vulnerabilidad ${ }^{4}$. En tercer lugar, los problemas de acceso al aborto derivados de la COVID-19 son, en gran parte de los casos y como se planteará más adelante, fácilmente resolubles, frente a las dificultades técnicas y logísticas que envuelven a otros procesos asistenciales (cirugías, revisiones oculares, tratamientos de fisioterapia, psicoterapia, odontológicos, etc.). Adicionalmente, la obstaculización que se está observando en lugares donde el aborto es legal no deriva directamente de la dificultad en la distribución de recursos generada por la pandemia; más bien parece que la pandemia ha servido de excusa para incrementar los obstáculos existentes antes de la crisis (Bateman y Kasztelan, 2020; EP, 2020; ONU, 2020). A modo de ejemplo y sin ánimo de exhaustividad, a continuación se muestran algunas de las respuestas proporcionadas en Estados Unidos y Europa.

En Estados Unidos, el cierre de las clínicas en las que se practican interrupciones del embarazo a raíz de las leyes conocidas como US TRAP (Targeted Regulation of Abortion Providers) está teniendo una grave repercusión en mujeres pertenecientes a minorías raciales, quienes ya se encontraban con dificultades antes de la COVID-19 para acceder a los servicios prestados en estos centros (Abrams, 2020; Solazzo, 2019). En el plano internacional, la Administración Trump reclamó oficialmente a la ONU la eliminación de las referencias a la salud sexual y reproductiva del Plan Global de Respuesta Humanitaria y la exclusión de la interrupción del embarazo como servicio esencial en la gestión de la COVID-19 (ONU, 2020).

En Polonia, uno de los países europeos más restrictivos en materia sexual y reproductiva, se aprobó en plena crisis pandémica un trámite para eliminar la posibilidad de abortar debido a anomalías o enfermedades incurables del feto, lo que condujo a centenares de mujeres a manifestarse en contra, a pesar del distanciamiento obligatorio para evitar contagios (Bateman y Kaztelan, 2020). El gobierno polaco ha optado por enviar esta iniciativa a una comisión parlamentaria, de modo que queda temporalmente suspendida. Mientras, en Rumanía, las interrupciones del embarazo solo están siendo atendidas en caso de urgencia, es decir, se reducen a aquellas que pueden considerarse como «prestación esencial» (EPF e IPPF, 2020). El resto se toma como una suerte de privilegio, "un lujo", que en condiciones de pandemia el sistema no se puede permitir (Tran et al., 2020).

A pesar de los datos anteriores, diversos organismos y organizaciones apelan a la necesidad de considerar el acceso al aborto como un servicio esencial. Por ejemplo, el Real Colegio de Obstetras y Ginecólogos, del Reino Unido, junto con otras instituciones, ha defendido el aborto como una prestación esencial de salud y la necesidad de que los servicios se organicen para proveer

4. Acerca del concepto de vulnerabilidad en el contexto específico de la pandemia, se recomienda consultar el artículo de Lydia Feito Grande, publicado en este mismo monográfico. 
de manera prioritaria una atención segura a las mujeres, incluyendo a aquellas que pudieran estar afectadas por la COVID-19 (RCOG, 2020).

Ante estas circunstancias, el denominado aborto medicamentoso resurge como una alternativa que ofrece un acceso seguro al aborto durante el primer trimestre de gestación (OMS, 2014), fuera de los espacios clínicos y con una menor sujeción a las restricciones legales (Calkin, 2019; Triviño, 2012). Además, supone un recurso para evitar desplazamientos innecesarios (Todd-Gher y Shah, 2020; Upadhyay, 2017), especialmente en una situación como la producida por la COVID-19, en la que el mandato imperante está siendo permanecer en casa y evitar lugares cerrados y concurridos que incrementen el riesgo de contagio.

En Francia, donde ya era posible el aborto farmacológico en el domicilio hasta las siete semanas, el plazo se ha extendido hasta las nueve semanas, aunque se planea revertir la medida una vez se alcance la fase de desconfinamiento (Elzas, 2020). En el Reino Unido, la COVID-19 ha dado lugar a la autorización del aborto farmacológico controlado a través de la telemedicina (Margolis, 2020). Las mujeres pueden realizar el tratamiento necesario para abortar en sus casas durante las diez primeras semanas de embarazo tras contactar telemáticamente con su médica o médico, sin necesidad de desplazarse a ningún centro sanitario. Esta medida no solo es coherente con las dificultades de acceso a las clínicas (Margolis, 2020) y con el escenario de aislamiento general prescrito durante la pandemia, sino que también permite que las mujeres puedan interrumpir sus embarazos con seguridad e intimidad, prescindiendo de intervenciones quirúrgicas innecesarias.

En España, la posibilidad de evitar desplazamientos para el asesoramiento obligatorio previo al acceso al aborto fue establecida como una competencia autonómica (Carballar, 2020). Solo Galicia y Cataluña han accedido a la propuesta de la Asociación de Clínicas Acreditadas para la Interrupción del Embarazo (ACAI) de sustituir el asesoramiento presencial por la modalidad telemática. En otras comunidades autónomas, como Madrid, las decisiones en relación con la interrupción de la gestación han tomado una dirección opuesta, como muestra el reciente reconocimiento de la personalidad jurídica del concebido, mediante la modificación de la Orden 340/2017 (Álvarez, 2020).

El aborto farmacológico, que en España es minoritario frente al quirúrgico y que ha de realizarse obligatoriamente en un centro autorizado, no se ha planteado como alternativa durante la pandemia. Esta situación puede haber supuesto un obstáculo importante para las mujeres en territorios donde no hay hospitales públicos ni clínicas que practiquen abortos, como es el caso de Ceuta y Melilla, o para aquellas mujeres cuyas responsabilidades de cuidado implican no poder salir de casa. Las restricciones de acceso resultan especialmente preocupantes para mujeres en situación de mayor vulnerabilidad, como aquellas que sufren violencia machista, confinadas con su maltratador; menores que requieren el consentimiento de sus padres o tutores para abortar, o migrantes indocumentadas (AAVV, 2020a). 
El tiempo es un factor determinante para que la interrupción del embarazo se realice con seguridad. Una atención puntual, segura y cuidadosa es fundamental para garantizar el bienestar y la salud de las mujeres. En el caso del aborto, su consideración como prestación esencial y la posibilidad de que pueda realizarse de forma ambulatoria es una reivindicación con una larga trayectoria que cobra especial relevancia en tiempos de pandemia. La crisis por COVID-19 no puede convertirse en una oportunidad para negar un derecho o convertirlo en un lujo, en un servicio prescindible, como se ha intentado (Bateman y Kasztelan, 2020; EP, 2020; ONU, 2020).

\section{El parto en tiempos de coronavirus ${ }^{5}$}

No queremos parir solas ${ }^{6}$.

The NICU experience is already stressful as it is, and then to force parents and babies to be separated-it's just devastating. How will babies [in NICUs] get the mothers' milk they so desperately need, if you're separating mothers and babies? (Testimonio de una matrona, en Furlow, 2020: 1)

«No queremos parir solas» es el lema empleado para una petición de firmas en Change.org, siguiendo el precedente de un caso análogo en Nueva York, dirigida a los servicios de obstetricia de hospitales españoles para evitar que ninguna mujer se vea obligada, a raíz de las crisis del COVID-19, a afrontar el proceso de parto sin el debido acompañamiento.

«Las matronas denuncian a la Generalitat Valenciana tras querer imponer que las embarazadas den a luz solas"; este es un titular del 4 de abril de este año de un diario español de tirada nacional (Martínez, 2020a). En efecto, el nuevo protocolo elaborado en dicho mes por la Consejería de Sanidad de la Comunidad Valenciana prescribía la interrupción del acompañamiento de la mujer gestante en dilatación y paritorios (AAVV, 2020b). A raíz de tales disposiciones se multiplicaron movilizaciones públicas en grupos diversos como el Consejo de Enfermería, la Associació de Comares, El Parto es Nuestro o la Asociación Española de Matronas, a las que se sumó la denuncia por atentar contra los derechos humanos, las propias leyes y, sobre todo, la evidencia científica publi$\mathrm{cada}^{7}$. Todo ello sucede en un ambiente ya caldeado por una condena pionera y recientísima de la $\mathrm{ONU}^{8}$ a nuestro país por violencia obstétrica contra una madre y su bebé, que motivó también una recomendación específica de la

5. Un anticipo sintético y divulgativo de estas reflexiones vio la luz en la prensa digital argentina (Massó Guijarro, 2020).

6. Cf. <https://www.change.org/p/jefes-y-jefas-de-servicio-de-obstetricia-y-ginecolog\% C3\%ADa-no-queremos-parir-solas $>$.

7. Ver referencias y notas a pie al respecto en la introducción.

8. Decisión adoptada por el Comité en virtud del artículo 4, párrafo 2 c), del Protocolo Facultativo, respecto de la comunicación núm. 138/2018, Comité para la Eliminación de la Discriminación contra la Mujer, de 28 de febrero de 2020. 
OMS a España por sus escandalosas cifras en este tipo de violencia de género (EPEN, 2020).

Por otro lado, la asombrosa celeridad y la efectividad de las asociaciones científicas y cívicas indujeron resultados sorpresivamente rápidos: apenas cuatro días después, la Generalidad Valenciana se retractó sobre las disposiciones más severas (Martínez, 2020b) y no mucho después la Junta de Andalucía publicó el Protocolo de acompañantes para la humanización durante el periodo de pandemia COVID-19, más acorde con las recomendaciones obstétricas y con los reclamos ciudadanos.

Numerosos autores han señalado las consecuencias negativas que las medidas derivadas de la distancia social y el confinamiento están comportando en mujeres y menores desde una perspectiva de género ${ }^{10}$, así como el impacto específico en general de la COVID-19 en las mujeres (AAVV, 2020c), concretamente en relación con los cuidados perinatales y la obstetricia. Buekens et al. (2020) destacan tales efectos adversos ligados al abandono de comportamientos de cuidado en el hogar (preventivos de tantas enfermedades) o al incremento de las depresiones posparto y otras dolencias psicológicas relacionadas, apelando también directamente a los efectos deletéreos de las restricciones vinculadas a la atención al parto y su negativo impacto psicológico para las mujeres aisladas.

Y es que se considera probado (Caparrós-González, 2020; Teti et al., 2020), en relación con el embarazo (la esfera perinatal en general) y la afectación de la COVID-19, la inexistencia de una mayor susceptibilidad a su infección en gestantes, así como de la transmisión vertical de SARS-CoV-2 de la madre al bebé, quien presenta un riesgo muy bajo de ser dañado por la infección (Teti et al., 2020). A mayor abundamiento se ha contrastado la ausencia de SARSCoV-2 en muestras de leche materna, líquido amniótico y sangre de cordón umbilical de recién nacidos de madres con COVID-19 (Caparrós-González, 2020). En la misma línea, el Grupo de Trabajo Interinstitucional sobre Salud Reproductiva en Situaciones de Crisis (McGovern et al., 2020; Tran et al., 2020) ha señalado, en diferentes guías para la salud sexual-reproductiva elaboradas en relación con la COVID-19 y bajo los auspicios de la evidencia científica, que las disposiciones de protección en momentos de parto y puerperio nunca deberían suponer un aumento de la violencia obstétrica. Así, se debería vigilar especialmente el incremento de cesáreas evitables o la limitación de la práctica «piel con piel» (un derecho humano también avalado científicamente $^{11}$ ) a través de separaciones innecesarias (Teti et al., 2020); también la

9. En este enlace se puede consultar el documento íntegro del CEDAW: <https://www.elparto esnuestro.es/sites/default/files/public/blog/cedaw-c-75-d-138-2018_spanish.pdf>.

10. Ver, por ejemplo, los datos del Global Health 50/50-COVID-19 sex-disaggregated data tracker: <http://globalhealth5050.org/covid19/>.

11. El poder estar en contacto «piel con piel» con la propia criatura y la propia madre (es una realidad mutua, evidentemente) tras el nacimiento no solo constituye un derecho humano relacional básico, sino que ha sido avalado científicamente de forma abrumadora desde hace décadas (Moore et al., 2016). Dicho de un modo contundente: limitar o entorpecer de 
falta de apoyo en el puerperio, que ya sabemos que afecta crucialmente a la salud materno-infantil, sobre todo en relación con la lactancia humana en sí misma, medida de salud pública fundamental a medio y largo plazo, entre otras implicaciones ahora inabordables (Massó Guijarro, 2015a y 2015b).

Respecto a la lactancia humana, dada su relación íntima con la esfera perinatal, el bienestar materno-infantil y el parto, conviene considerar que las expertas han destacado cómo las disrupciones en la lactancia durante la pandemia incrementan el riesgo de los bebés de sufrir enfermedades agravadas (Furlow, 2020). Desde la Red colaborativa virtual de bancos y asociaciones de leche materna se ha reseñado la importancia de mantener la provisión segura de leche humana a través de la donación a los bancos, a causa de su mayor relevancia por el contexto pandémico (Shenker, 2020). Se incide además en que el SARS-CoV-2 no se transmite por la leche humana y que la lactancia resulta más necesaria que nunca, sobre todo en grandes prematuros y bebés con anomalías congénitas o trastornos neurológicos. Por todo ello, la OMS (2020) ha elaborado documentación específica para asesorar acerca de la lactancia en la presente pandemia ${ }^{12}$.

Sobre la conveniencia de la separación de la madre y el neonato, destaca el análisis de Teti et al. (2020), que apunta a la necesidad de una autonomía decisional informada en la madre y/o en los progenitores. Las decisiones unilaterales de separación, a tenor de las negativas consecuencias de diverso tipo que implican, se desaconsejan absolutamente. La pauta se orienta a reservar las separaciones para casos graves indicados, siempre y cuando la madre acepte esta medida en última instancia, amparándose su decisión última en todo momento y apoyándola de modo práctico para hacerla viable (por ejemplo, en relación con la lactancia, tanto si es en diferido, en caso de separación, como si es directa).

\section{Conclusiones}

La pandemia por COVID-19 ha obligado a redefinir e, incluso, restringir lo que puede considerarse "cuidado esencial» en tiempos de crisis. En ese sentido, la atención a la salud sexual, reproductiva y puerperal se ha visto amenazada y convertida en un objetivo de debate. Por un lado, el derecho de las mujeres a elegir libremente cómo parir y cómo abortar implica garantizar su acceso a una prestación de estos servicios basada en la evidencia científica y disponible

cualquier forma, sin un motivo muy poderoso, el contacto directo que ya es proverbial llamar de "piel con piel» entre madre y criatura tras el parto supone un compromiso tan importante de la salud y el bienestar de ambas, especialmente de la segunda, pero también de la primera, que dificultarlo, no informarlo o no favorecerlo en primera instancia entra sin ambages dentro de los parámetros de la violencia obstétrica.

12. Groß et al. (2020) sí han detectado, muy recientemente, presencia de SARS-CoV-2 en la leche humana en un caso aislado, aunque afirman que no está claro si la transmisión al recién nacido (por otro lado, inofensiva) fue por esta o por otra vía. En general, se mantiene el consenso de la altísima conveniencia de amamantar. 
para todas, proporcionando especial atención a aquellas que se encuentren en situación de vulnerabilidad por distintas razones (situación administrativa, personal, familiar, laboral; falta de información y recursos; etc.). Por otro lado, urge atender los efectos indirectos que la pandemia ha supuesto para la mortalidad materno-infantil a nivel global, así como las consecuencias negativas de las condiciones del confinamiento para la infancia (Fore, 2020), exponente de un sistema adultocéntrico y patriarcal.

Como ya se apuntó, se podría argumentar o cuestionar si la pérdida de protección de la salud sexual y reproductiva de las mujeres se debe a un sesgo de género, al patriarcado en definitiva, o a otras causas. Ello podría acaso presentar visos de verosimilitud si no existiera la violencia obstétrica reconocida como tal - hasta el punto de que incluso la COVID-19 ya ha sido catalogada literalmente como "factor de riesgo" para dicha violencia obstétrica (Sadler et al., 2020)_, de un lado, y los datos incontrovertibles sobre el impacto trágico de la pandemia en mujeres y niños (Roberton et al., 2020; Menendez et al., 2020), de otro. Esto es el patriarcado. "Cuando dos elefantes pelean, es la hierba la que sufre», afirma un proverbio africano que ha llegado a constituir sabiduría globalizada desde su popularización durante los procesos de descolonización en África en la década de 1960.

Cuando hay una pandemia mundial, el prisma de la interseccionalidad ${ }^{13}$ nos ayuda a comprender por qué es «la hierba» (mujeres gestantes y puérperas, menores) la que sufre (más).

Lejos de aprovechar la situación de emergencia para restringir y negar cuidados relacionados con el parto y el aborto a partir de premisas ideológicas, la COVID-19 puede convertirse en una oportunidad para revisar y cuestionar prejuicios e inercias institucionales sobre el proceso de parto, las prácticas ligadas al vínculo materno-filial, la necesidad de realizar trámites como el asesoramiento obligatorio previo al aborto o su modalidad farmacológica fuera de los centros sanitarios. De ese modo, será posible afianzar los derechos de las mujeres y la primera infancia, que, lejos de ser un lujo, constituyen una condición necesaria para la igualdad.

\section{Referencias bibliográficas}

AAVV (2020a). Joint Civil Society Statement European governments must ensure safe and timely access to abortion care during the COVID-19 pandemic. Recuperado de <https://www.hrw.org/news/2020/04/08/joint-civilsociety-statement-european-governments-must-ensure-safe-and-timely>.

13. Para ahondar en otros rostros de los tantos que señala este prisma en relación con las diferentes formas de discriminación y su intrincado entrecruzamiento, y tal como se muestra también en la introducción (editorial) de este mismo monográfico, véanse en él los artículos de Jimena Mantilla (para el caso de la infancia y el adultocentrismo), de Jon Rueda (para el caso del edadismo y la gerontofobia) y de Soledad Arnau (para el caso de la diversidad funcional). 
- (2020b). Manejo de la mujer embarazada y el recién nacido con COVID-19. Documento técnico del Ministerio de Sanidad del Gobierno de España, versión de 17 de marzo de 2020.

- (2020c). Policy Brief: The Impact of COVID-19 on Women. Naciones Unidas. Global Humanitarian Response Plan Covid-19 United Nations Coordinated Appeal April-December 2020. Recuperado de <https://www. unocha.org/sites/unocha/files/Global-Humanitarian-Response-PlanCOVID-19.pdf $>$.

Aвrams, Abigail (2020). «COVID-19 could permanently make abortions harder to access nationwide». Time (7 de abril). Recuperado de <https:// time.com/5816530/coronavirus-abortion-clinics-access/>.

Almaguer GonzÁlez, José Alejandro et al. (2010). «La violencia obstétrica: Una forma de patriarcado en las instituciones de salud». Género y Salud en Cifras, 8 (3), 4-20.

Álvarez, Ángeles (2020). La Comunidad de Madrid se salta principios juridicos en su estrategia contra el aborto legal. Recuperado de <https://tribunafemi nista.elplural.com/2020/04/la-comunidad-de-madrid-se-salta-principiosjuridicos-en-su-estrategia-contra-el-aborto-legal/>.

Bateman, Jessica y Kasztelan, Marta (2020). «In Poland, Abortion Access Worsens Amid Pandemic». Foreign Policy (1 de mayo). Recuperado de $<$ https://foreignpolicy.com/2020/05/01/poland-abortion-access-worsenscoronavirus-pandemic/>.

Buekens, Pierre et al. (2020). «A call for action for COVID-19 surveillance and research during pregnancy». Lancet Global Health, 8 (7), E877-E878. <https://doi.org/10.1016/S2214-109X(20)30206-0>

Calkin, Sydney (2019). «Towards a political geography of abortion». Political Geography, 69, 22-29.

Campbell, Rona et al. (1997). «Feminist theory and the sociology of childbirth: A response to Ellen Annandale and Judith Clark». Sociology of Health \& Illness, 9 (13), 348-358. <https://doi.org/10.1111/j.1467-9566.1997.tb00023.x>

Caparrós-GonzÁlez, Rafael (2020). "Consecuencias maternas y neonatales de la infección por coronavirus COVID-19 durante el embarazo: Una scoping review». Revista Española de Salud Pública, 94, e202004033.

Carballar, Olivia (2020). Sanidad deja la decisión sobre los trámites para abortar en manos de las CCAA (8 de abril). Recuperado de <https://www. lamarea.com/2020/04/08/sanidad-deja-la-decision-sobre-los-tramitespara-abortar-en-manos-de-las-ccaa/>.

ElzAS, Sarah (2020). France extends access to abortions during Covid-19 pandemic (11 de abril). Recuperado de <http://www.rfi.fr/en/france/20200411france-extends-access-to-abortions-during-covid-19-pandemic>.

EP (Europa Press) (2020). El Supremo rechaza cerrar de urgencia las clínicas que practican abortos en el estado de alarma (4 de mayo). Recuperado de $<$ https://www.europapress.es/nacional/noticia-supremo-rechaza-cerrarurgencia-clinicas-practican-abortos-estado-alarma-20200504163135.html>. 
EPEN (2020). «Condena histórica: La ONU condena al estado español y le exige combatir la violencia obstétrica». El parto es nuestro. Recuperado de <https:// www.elpartoesnuestro.es/blog/2020/03/09/condena-historica-la-onu-conde na-al-estado-espanol-y-le-exige-combatir-la-violencia-obstetrica> .

EPF (European Parliamentary Forum for Sexual and Reproductive Rights) e IPPF (International Planned Parenthood Federation) (2020). Sexual and Reproductive Health and Rights during the COVID-19 pandemic (22 de abril). Recuperado de <https://www.ippfen.org/sites/ ippfen/files/2020-04/Sexual\%20and\%20Reproductive\%20Health\%20 during\%20the \%20COVID-19\%20pandemic.pdf>.

Fore, Henrietta H. (2020). «A wake-up call: COVID-19 and its impact on children's health and wellbeing». The Lancet Global Health, 8 (7), E861-E862. $<$ https://doi.org/10.1016/S2214-109X(20)30238-2>

Furlow, Bryant (2020). "US NICUs and donor milk banks brace for COVID-19». Lancet Child Adolescent Health, 4 (5), P355. $<$ https://doi.org/10.1016/S2352-4642(20)30103-6>

Gross, Rüdiger et al. (2020). "Detection of SARS-CoV-2 in human breastmilk». The Lancet Digital Health, 395 (10239), P1757-1758. <https://doi.org/10.1016/S0140-6736(20)31181-8>

GutTMaCher Institute (2020). The COVID-19 Outbreak: Potential Fallout for Sexual and Reproductive Health and Rights (11 de mayo). Recuperado de <https://www.guttmacher.org/article/2020/03/covid-19-outbreakpotential-fallout-sexual-and-reproductive-health-and-rights>.

Hussein, Julia (2020). "COVID-19: What implications for sexual and reproductive health and rights globally?». Sexual and Reproductive Health Matters, 28 (1). <https://doi.org/10.1080/26410397.2020.1746065>

IPPF (International Planned Parenthood Federation) (2020). Declaración del IMAP sobre el COVID-19 y su impacto en la salud sexual y reproductiva (abril de 2020). Recuperado de <https://www.ippf.org/sites/ default/files/2020-04/IMAP\%20Statement\%20on\%20COVID-19\%20 impact\%20on\%20SRHR\%20-\%20Spanish.pdf>.

MARGOLIS, Hillary (2020). England Leads Way in UK after U-Turn on COVID19 Abortion Access (31 de marzo). Recuperado de <https://www.hrw.org/ news/2020/03/31/england-leads-way-uk-after-u-turn-covid-19-abortionaccess $>$.

MartíneZ, Ana (2020a). «Las matronas denuncian a la Generalitat Valenciana tras querer imponer que las embarazadas den a luz solas». $A B C$ (4 de abril). Recuperado de $<$ https://www.abc.es/familia/mujeres/abci-embarazo202004061251_noticia.html?ref=https\%3A\%2F\%2Fwww.google. com $\% 2 \mathrm{~F}>$.

- (2020b). «Las matronas consiguen que la Generalitat Valenciana recule en su idea de que las embarazadas den a luz solas». $A B C$ (8 de abril). Recuperado de $<$ https://www.abc.es/familia/mujeres/abci-matronas-consiguen-generali tat-valenciana-recule-idea-embarazadas-solas-202004080918_noticia.html>. 
Massó Gujjarro, Ester (2015a). «Lactivismo contemporáneo en España: ¿Una nueva marea sociopolítica?». Journal of Spanish Cultural Studies, 1 (16), 193-213. $<$ https://doi.org/10.1080/14636204.2015.1069074>

- (2015b). "Una etnografía lactivista: La dignidad lactante a través de deseos y políticas». Revista de Antropología Iberoamericana, 10 (2), 231-257. $<$ https://doi.org/10.11156/aibr.100205>

- (2020). «Malos tiempos para parir: Coronavirus y violencia obstétrica». El País Digital (21 de junio). Recuperado de <https://www.elpaisdigital.com.ar/con tenido/malos-tiempos-para-parir-coronavirus-y-violencia-obsttrica/ 27350>.

McGovern, Terry et al. (2020). "Centring sexual and reproductive health and justice in the global COVID-19». The Lancet, 395, 1175-1177.

Menendez, Clara et al. (2020). "Avoiding indirect effects of COVID-19 on maternal and child health». The Lancet Digital Health (12 de mayo). <https://doi.org/10.1016/S2214-109X(20)30239-4>

Moore, Elizabeth et al. (2016). «Early skin-to-skin contact for mothers and their healthy newborn infants». Cochrane Database of Systematic Reviews, 11 , art. n. ${ }^{\circ}$ CD003519. <https://doi.org/10.1002/14651858.CD003519.pub4>

OMS (2014). Clinical practice handbook for safe abortion. Recuperado de <https:// apps.who.int/iris/bitstream/handle/10665/97415/97892415 48717_eng.pdfs.

- (2020). Campaigns - Breastfeeding advice during COVID-19. Recuperado de <http://www.emro.who.int/noncommunicable-diseases/campaigns/ breastfeeding-advice-during-the-covid-19-outbreak.html>.

ONU (2020). Autoridades en Estados Unidos manipulan la crisis del coronavirus para restringir el acceso al aborto, dicen expertos de la ONU. Recuperado de <https://news.un.org/es/story/2020/05/1475022>.

Rámila Gómez, María et al. (2016). «Importancia del acompañamiento continuo en cesáreas programadas: Ansiedad materna». Biblioteca Lascasas, 12 (3), 9 y s. Recuperado de <http://www.index-f.com/lascasas/documentos/ lc0919.php>.

RCOG (Royal College of Obstetrics and Gynecology) (2020). Guidance for healthcare professionals on coronavirus (COVID-19) infection and abortion care. Recuperado de <https://www.rcog.org.uk/globalassets/ documents/guidelines/2020-06-03-coronavirus-covid-19-infection-andabortion-care.pdf $>$.

Roberton, Timothy et al. (2020). «Early estimates of the indirect effects of the COVID-19 pandemic on maternal and child mortality in low-income and middle-income countries: A modelling study». The Lancet, 8 (7), E901-E908. <https://doi.org/10.1016/S2214-109X(20)30229-1>

Robinson, Erica F. et al. (2020). «Preserving and advocating for essential care for women during the coronavirus disease 2019 pandemic». American Journal of Obstetrics and Gynecology, 223 (2), 219-220. <https://doi.org/10.1016/j.ajog.2020.05.022> 
Ross, Loretha (2017). «Reproductive Justice as Intersectional Feminist Activism». Souls, 19 (3), 286-314. <https://doi.org/10.1080/10999949.2017.1389634>

SADLER, Michelle et al. (2020). "COVID-19 as a risk factor for obstetric violence». Sexual and Reproductive Health Matters, preprint. <https://doi.org/10.1080/26410397.2020.1785379>

Sethna, Christabelle y Davis, Gayle (eds.) (2019). Abortion across borders: Transnational travel and access to abortion services. Baltimore: John Hopkins University Press.

SHENKer, Natalie (2020). «Maintaining safety and service provision in human milk banking: A call to action in response to the COVID-19 pandemic». Lancet Child Adolescent Health, 4 (7), P484-485. <https://doi.org/10.1016/S2352-4642(20)30134-6>

Solazzo, Alexa L. (2019). «Different and not equal: The uneven association of race, poverty, and abortion laws on abortion timing». Social Problems, 66, 519-547.

Tarodo Soria, Salvador y GonzÁlez de Zárate, Javier (2012). «Responsabilidad del profesional de la sanidad en la atención al parto natural o normal». En: GoNZÁLEZ DE ZÁrATE, Javier et al. Estrategia para la implantación del parto normal: Cursos para matronas, obstetras y anestesiólogos. Valladolid: González de Zárate.

Teti, Stowe Locke et al. (2020). «Should New Mothers With Covid-19 Be Separated From Their Newborns?». Hastings Bioethics Forum (11 de mayo). Recuperado de <https://www.thehastingscenter.org/why-new-motherswith-covid-19-should-not-be-separated-from-their-newborns/>.

Todd-Gher, Jaime y Shah, Payal K. (2020). "Abortion in the context of COVID-19: A human rights imperative». Sexual and Reproductive Health Matters, 28 (1).

$<$ https://doi.org/10.1080/26410397.2020.1758394>

Tran, Nguyen Toan et al. (2020). «Not a luxury: A call to maintain sexual and reproductive health in humanitarian and fragile settings during the COVID-19 pandemic». Lancet Global Health, 8 (6), E760-E761. $<$ https://doi.org/10.1016/S2214-109X(20)30190-X>

Triviño Caballero, Rosana (2012). «Mujeres migrantes y misoprostol: Aborto privado, escándalo público». Dilemata, 10, 31-44.

- (2018). "Acciones indebidas en el acceso al aborto: A propósito del periodo de espera y asesoramiento obligatorios». En: TRIviño, Rosana y Ausín, Txetxu Ausín (eds.). Hacer o no hacer: La responsabilidad por acciones y omisiones. Madrid: Plaza y Valdés.

UpADHYAY, Ushma D (2017). "Innovative models are needed for equitable abortion access in the USA». The Lancet Public Health, 2 (11), e484-e485.

Watson, Katie (2018). "Why We Should Stop Using the Term "Elective Abortion"». AMA Journal of Ethics, 20 (12), e1175-e1180. $<$ https://doi.org/10.1001/amajethics.2018.1175> 
Ester Massó Guijarro es profesora titular de Filosofía Moral en el Departamento de Filosofía I de la Universidad de Granada. Miembro de ESPACyOS (Ética Salubrista para la Acción, Cuidado y Observación Social) y de la Unidad de Excelencia Docente «FiloLab». Profesora en el Departamento de Antropología Social de la UGR, adscrita a la misma área entre 2012 y 2019. Doctora (2009), licenciada en Filosofía y en Antropología Social y Cultural (2003) por la Universidad de Granada y máster en Cooperación Internacional por las universidades de Granada y de Santiago de Compostela (2005). Ha realizado estancias de investigación en el Instituto de Ciencias Sociales de la Universidad de Lisboa (Portugal), la Universidad de Windhoek (Namibia), el Instituto Nórdico de África de Uppsala (Suecia) y el Instituto de Ciencias Antropológicas de la Universidad de Buenos Aires (Argentina). Dos sexenios de investigación reconocidos por el CNAI. Investigadora predoctoral (FPU) en la UGR y posdoctoral (JAE DOC) en el CSIC (Madrid).

Ester Massó Guijarro is a professor of moral philosophy in the Department of Philosophy I at the University of Granada (UGR). She is a member of the ESPACyOS network on Health Ethics for Action, Care and Social Observation and the FiloLab Unit of Teaching Excellence. From 2012 to 2019, she was a professor of anthropology in the Department of Social Anthropology at the UGR. She holds a PhD (2009), a Licentiate of Philosophy and Social \& Cultural Anthropology (2003) from the UGR and a Master of International Cooperation from the UGR and the University of Santiago de Compostela (2005). She has done several research stays at the Institute of Social Sciences of the University of Lisbon, Portugal; the University of Windhoek in Namibia; the Nordiska Afrikainstitutet of Uppsala, Sweden and the Institute of Anthropological Sciences of the University of Buenos Aires, Argentina. She has also been a predoctoral researcher at the UGR and a postdoctoral researcher (JAE DOC) at the Spanish National Research Council (CSIC) of Madrid.

Rosana Triviño Caballero es profesora ayudante doctora en la Facultad de Medicina y Ciencias de la Salud de la Universidad de Alcalá. Miembro de ESPACyOS (Ética Salubrista para la Acción, Cuidado y Observación Social) y de LI²FE (www.liife.org). Doctora en Filosofía (USAL, 2014), licenciada en Humanidades (UC3M, 2007) y diplomada en Fisioterapia (UPCO, 1998). Obtuvo el diploma en Ciencia Política y Derecho Constitucional (CEPC, 2009), cuyo trabajo final sobre autonomía del paciente y rechazo del tratamiento por motivos religiosos recibió el premio al mejor trabajo de investigación. Ha sido investigadora JAE-predoctoral en el Instituto de Filosofía del CSIC y ha realizado estancias de investigación en Oxford (Centro Uehiro de Ética Aplicada), Cleveland (Universidad Case de la Reserva Occidental), Princeton (Universidad de Princeton) y Nueva York (Centro Hastings). En 2017, obtuvo una beca de investigación en bioética de la Fundación Grífols para financiar el proyecto ProtoAccess sobre las condiciones de acceso de la IVE en la UE.

Rosana Triviño Caballero is assistant lecturer at the Faculty of Medicine and Health Sciences of Alcalá University, Madrid. She is member of the ESPACyOS network on Health Ethics for Action, Care and Social Observation and LI²FE (www.liife.org). She holds a PhD in Philosophy from the University of Salamanca (USAL, 2014), a Licentiate of Humanities from the Carlos III University of Madrid (UC3M, 2007) and a Bachelor of Physiotherapy from the Comillas Pontifical University (UPCO, 1998). She earned a Master of Political Science and Constitutional Law at the Center for Political and Constitutional Studies (CEPC, 2009) for which she was awarded the prize for outstanding research for her work on patient autonomy and treatment refusal grounded on religious beliefs. She has been a predoctoral researcher at the Institute of Philosophy of the Spanish National Research Council (CSIC). She has also done several research stays at the Uehiro Center for Applied Ethics, University of Oxford; Case Western Reserve University of Cleveland, Ohio; Princeton University and The Hastings Center of New York. In 2017, she was awarded a bioethics research grant from the Grífols Foundation to fund the ProtoAccess Project on protocols for accessing pregnancy interruption in the EU. 